\title{
Avaliação do potencial antimicrobiano do extrato etanólico bruto e frações da Sida santaremnensis H. MONTEIRO (MALVACEAE) sobre cepas de Rhodotorula spp.
}

\section{Antimicrobial potential of the evaluation of the ethanol extract and fractions of Sida santaremnensis H. MONTEIRO ( MALVACEAE ) on strains of Rhodotorula spp.}

\author{
Josefa Paula do Nascimento ${ }^{1}$; Danielly Albuquerque da Costa ${ }^{2}$; Edeltrudes de Oliveira Lima ${ }^{3}$ Maria Carmem Batista de \\ Alencar ${ }^{4}$ e Egberto Santos Carmo ${ }^{2}$.
}

\begin{abstract}
RESUMO - As leveduras do gênero Rhodotorula estão associadas a uma variedade de processos patológicos no homem, nos casos mais severos a taxa de mortalidade chega a 50\%. A anfotericina B e o fluconazol são os fármacos de escolha utilizados no tratamento de infecções por Rhodotorula spp. no entanto, algumas cepas dessas leveduras já apresentam graus de resistência ao fluconazol. Por apresentarem diferentes propriedades terapêuticas e relatos de uso, as plantas do gênero Sida (Malvaceae) vêm sendo amplamente estudadas. A espécie Sida santaremnensis ocorre em quase todo o Brasil, apesar de sua ampla distribuição, não há relato de utilização dessa espécie na medicina popular. Contudo, verificando a variedade de compostos naturais, propriedades farmacológicas e relatos de uso descritos para espécies da família Malvaceae, notadamente no gênero Sida, e, visando contribuir para o desenvolvimento de novos antifúngicos, buscou-se avaliar através da determinação da Concentração Inibitória Mínima (CIM) e da Concentração Fungicida Mínima (CFM) a atividade antimicrobiana do extrato etanólico bruto (EEB) e das frações hexânica (HEX), diclorometânica $\left(\mathrm{CH}_{2} \mathrm{Cl}_{2}\right)$, acetato de etila (AcOEt) e hidroalcoólica (ETOH: $\mathrm{H}_{2} \mathrm{O}$ ) de $S$. santaremnensis sobre doze cepas de Rhodotorula spp. A determinação da CIM de S. santaremnensis foi realizada pela técnica de microdiluição, utilizando placas com 96 cavidades. Após realização da microdiluição determinou-se a CFM. Os ensaios foram realizados em duplicata e o resultado expresso pela média geométrica das concentrações inibitórias obtidas nos dois ensaios. Verificou-se que o EEB e as frações $\mathrm{CH}_{2} \mathrm{Cl}_{2}$ e AcOEt inibiram três das doze cepas testadas. A CIM, onde não se visualizou crescimento fúngico, esteve entre $2048 \mu \mathrm{g} / \mathrm{mL}$ e $512 \mu \mathrm{g} / \mathrm{mL}$. O extrato e frações que apresentaram ação antifúngica, também demonstraram comportamento fungicida. A grande maioria das cepas de Rhodotorula spp. não foi sensível ao extrato etanólico bruto e frações de $S$. santaremnensis, demonstrando que o desenvolvimento de antifúngicos a base dessa planta, no futuro, não seria promissor para tratamento de infecções por Rhodotorulla spp.
\end{abstract}

Palavras-chaves: Rhodotorula spp., Sida santaremnensis, antifúngicos, microdiluição.

ABSTRACT - Rhodotorula is associated with a variety of pathological processes in humans, in severe cases the mortality rate reaches $50 \%$. The amphotericin B and fluconazole are the drugs of used in the treatment of infections Rhodotorula spp. however, some strains of the yeast already have degrees of resistance to fluconazole. The species Sida santaremnensis occurs in almost all Brazil, despite its wide distribution, there is no report of this kind of use in folk medicine. However, checking the variety of natural compounds, pharmacological properties and use reports to described species of the family Malvaceae, notably in the gender Sida, and to contribute to the development of new antifungal agents, we sought to assess by determining the antimicrobial activity by Minimum Inhibitory Concentration (MIC) and Minimum Fungicidal Concentration (CFM) of crude ethanol extract (BSE) and fractions of hexane (HEX), dichloromethane $\left(\mathrm{CH}_{2} \mathrm{Cl}_{2}\right)$, ethyl acetate (EtOAc) and wateralcohol (ETOH: $\left.\mathrm{H}_{2} \mathrm{O}\right)$ of $S$. santaremnensis about twelve strains Rhodotorula spp. The MIC determination of S. santaremnensis was performed by the microdilution technique, using 96 well plates. After performing microdilution determined to CFM. Assays were performed in duplicate and the results expressed as the geometric mean of the inhibitory concentrations obtained in two trials. It was found that the $\mathrm{BSE}$ and $\mathrm{CH}_{2} \mathrm{Cl}_{2}$ and EtOAc fractions inhibited three of the twelve strains tested. The MIC, where not visualized fungal growth was between $2048 \mu \mathrm{g} / \mathrm{mL}$ and $512 \mu \mathrm{g} / \mathrm{mL}$. The extract and fractions that showed antifungal activity, also were behaved fungicide. The majority of strains of Rhodotorula spp. was not sensitive to the crude extract and fractions of $S$. santaremnensis, demonstrating that the development of antifungal the basis of this plant in the future, it would not indicated for treatment of infections Rhodotorulla spp.

Key words: Rhodotorula spp., Sida santaremnensis, antifungal, microdilution.

\footnotetext{
*Autor para correspondência

Recebido para publicação em 06/10/2014; aprovado em 22/05/2015

${ }^{1}$ Bacharelado em Farmácia, da Unidade Acadêmica de Saúde, do Centro de Educação e Saúde, Universidade Federal de Campina Grande, campus Cuité/PB.

${ }^{2}$ Docente do Bacharelado em Farmácia, da Unidade Acadêmica de Saúde, do Centro de Educação e Saúde, Universidade Federal de Campina Grande, campus Cuité/PB.

${ }^{3}$ Docente do Bacharelado em Farmácia, Departamento de Ciências Farmacêuticas, Centro de Ciências da Saúde, Universidade Federal da Paraíba, João Pessoa/PB.

${ }^{4}$ Prof. da Faculdade São Francisco da Paraíba, FASP - Cajazeiras - PB Brasil e Mestranda do Curso de Pós graduação em sistemas agroindustriais do CCTA/UFCG/Pombal - PB. E-mail: carmemsjp@hotmail.com
} 


\section{INTRODUÇÃO}

O surgimento de patógenos fúngicos menos comuns vem sendo cada vez mais relatados nas últimas décadas. As leveduras do gênero Rhodotorula são capazes de habitar o organismo humano, elas estão associadas a uma variedade de processos patológicos no homem, frequentemente são encontradas como contaminante da pele, unhas, pulmão, urina, fezes, sistema nervoso central e sangue (MENDES et al., 2008; RICHARDSON; LASS-FLOR, 2008).

Classificada como uma infecção fúngica oportunista apresenta como fatores de risco para o seu desenvolvimento, a utilização de cateter venoso central (CVC), longos períodos de internações e doenças consideradas predisponentes, como as linfoproliferativas, a insuficiência renal crônica, o diabetes mellitus, a síndrome da imunodeficiência adquirida (SIDA) e outros. As leveduras do gênero Rhodotorula apresentam baixa virulência, mas, é na clínica que a fungemia causada por este agente normalmente possui comportamento agressivo, dentre os sinais clínicos comumente apresentados por esse tipo de infecção destacam-se febre, calafrios, taquicardia e letargia (RUIZ; ZORRILLA, 2001).

Nos casos mais severos a taxa de mortalidade chega a 50\%, na farmacoterapia utilizam-se os fármacos anfotericina B e o fluconazol, no entanto algumas cepas do gênero Rhodotorula já demonstraram certa resistência ao fluconazol (RUIZ; ZORRILLA, 2001; RANG; DALE, 2007; RICHARDSON; LASS-FLOR; 2008).

Espécies do gênero Sida encontram-se bastante representadas nas Américas, no Brasil são abundantes nas regiões Nordeste e Sul. Apresentam como característica comum a presença de fitoecdisteróides, composto responsável pela maioria de suas atividades biológicas, tais como tonificante, anabolizante, diurético e regulador de índices glicêmicos (LEAL, 2008).

Estudos revelam que a espécie Sida santaremnensis, popularmente conhecida como "vassourinha" ou "guanxuma" apresenta efeitos antiulcerogênicos (OLIVEIRA et al., 2008), antinociceptivos (MENDES et al., 2008), vasorelaxantes (ARCANJO et al., 2011; OLIVEIRA et al., 2011) e atividade antiendematogênica (SILVA, 2008; MOURA, 2010). Evidenciando desta forma, a possibilidade de realizarem-se pesquisas que venham a desvendar novos compostos bioativos dessa espécie, principalmente no tocante do potencial antimicrobiano.

Os fungos são seres ubíquos, estando presente em ambientes como água, terra, ar ou em parasitismo (LACAZ et al., 2002; ALMEIDA, 2008; SIDRIM; ROCHA, 2010). São organismos eucarióticos, classificam-se como seres heterotróficos, uma vez que aproveitam a energia contida nas ligações químicas de vários nutrientes. Algumas estruturas fúngicas podem ser visualizadas macroscopicamente, entretanto para a grande maioria delas a utilização do microscópio se faz necessária (SIDRIM; ROCHA, 2010).

Estruturas relacionadas à reprodução, principalmente sexuada revestem-se de importância capital, visto que nesses achados repousam os fundamentos da classificação taxonômica dos fungos, contudo quando à forma de reprodução sexuada não é detectada, a classificação é baseada nos órgãos de reprodução assexuada (SIDRIM; ROCHA, 2010).
Primariamente, os fungos são observados pela sua forma vegetativa. Sendo esta unicelular, como são conhecidas as leveduras, ou multicelular, no caso dos filamentosos (mais abundantes na natureza). Ainda existem os dimórficos, apresentam-se leveduriformes a temperatura de $37^{\circ} \mathrm{C}-39^{\circ} \mathrm{C}$ ou filamentosos a temperatura ambiente. Por serem dispersos no ambiente os fungos podem desencadear inúmeras doenças no organismo humano (LACAZ et al., 2002).

Os fungos são capazes de colonizar o homem e animais, mas quando ocorre um desequilíbrio entre o binômio parasito-hospedeiro, podem desencadear diversos quadros infecciosos com formas clínicas localizadas ou disseminadas (ANVISA, 2004). A gravidade da infecção fúngica varia desde formas cutâneas leves até quadros mais graves como septicemia, que geralmente culminam com a morte (LIMA et al., 2005).

As micoses podem ser divididas em micoses superficiais, como a Pitiríase vesicolor, Piedra branca e Piedra negra; cutâneas abrangendo as Dermatofitoses dentre elas a Tinea corporis; subcutâneas como a Cromoblastomicose, Esporotricose e os Micetomas; profundas, responsáveis pela Paracoccidioidomicose e Histoplasmose, e por fim, as oportunistas Candidíase, Criptococose e Aspergilose (SIDRIM; ROCHA, 2010).

Adequam-se ainda dentro da classe dos fungos oportunistas, as leveduras do gênero Rhodotorula. Estas são simbiontes de pele úmida, escarro, urina e fezes. São amplamente distribuídas na natureza, sendo isoladas do solo, do ar, da água, dos laticínios, dentre outros. Os casos de fungemias oriundas dessas leveduras originam-se principalmente devido à sua colonização em cateteres, soluções intravenosas, aparelhos e material cirúrgico contaminado (LACAZ et al., 2002).

Anteriormente eram consideradas como nãopatogênicas, no entanto durante as últimas duas décadas, elas emergiram como agente etiológico oportunista, principalmente em pacientes imunocomprometidos (TUON; COSTA, 2008; MICELI; DÍAZ; LEE, 2011).

As leveduras do gênero Rhodotorula, pertencem à família Sporidiobolaceae, da ordem Sporidiales. Este gênero possui trinta e quatro espécies sendo três delas relacionadas a infecções no homem, a saber: $R$. mucilaginosa, $R$. glutinis e $R$. minuta. $R$. mucilaginosa, nomenclatura atual de espécies previamente designadas $R$. rubra é a espécie mais frequentemente implicada nas diferentes infecções descritas no homem, seguida por $R$. glutinis (ALMEIDA, 2005). Os tipos de infecções comumente desencadeadas por essas leveduras são as do sistema nervoso central (meningite e ventriculites), as oculares (endoftalmite e ceratite) e as endocardites e peritonites (TUON; COSTA, 2008).

Estudos epidemiológicos mostram que Rhodotorula spp. é o agente etiológico em 0,5 a $2,3 \%$ dos casos de fungemia. Os principais fatores relacionados a isso são a utilização indiscriminada de antibióticos, prolongadas administrações de nutrição parenteral, devido aos casos de desnutrição secundária em doenças crônicas, onde na sua maioria ocorrem em casos de neoplasias, seguida por transplantes de medula óssea e SIDA. A mortalidade causada nos achados de fungemias por Rhodotorula spp. chega a 14,4\% (TUON; COSTA, 2008). 
O diagnóstico de leveduroses, como a Rhodotorula spp. baseia-se nas características clínicas da fungemia (inflamação, supuração, resposta granulomatosa), na morfologia de cada microrganismo através de exame direto, oriundo de raspados de lesões cutâneas e mucosa, sedimentos de escarro, urina, fezes, líquido cefalorraquidiano e lavado brônquicos, e na cultura das espécies, buscando conhecer o perfil bioquímico dos organismos (NEUFELD, 1999).

Suas colônias são detectáveis visualmente após 24 a 48 horas de incubação, geralmente apresentando aspecto liso e/ou mucoso, e pigmento carotenoide típico, variando de cor amarelada a avermelhada; a temperatura máxima de crescimento encontra-se entre $25^{\circ} \mathrm{C}$ e $37^{\circ} \mathrm{C}$ (KWONCHUNG; BENNETT, 1992; FELL; STATZELLTALLMAN, 2000 apud ALMEIDA, 2005).

Quanto à identificação das suas espécies, podem ser utilizadas técnicas automatizadas, por meio do sistema Vitek (bioMérieux, EUA), bem como técnicas manuais através do sistema APIC 20C AUX (bioMérieux, EUA). E ainda pode-se valer do sistema convencional manual de assimilação de carboidratos e nitritos, onde do ponto de vista bioquímico essas leveduras não fermentam carboidratos, não assimilam inositol e são urease positiva. As características microscópicas do gênero incluem: células leveduriformes ovais, esferoidais ou alongadas, com brotamento polar ou multilateral; ausência ou formação de pseudo-hifa ou hifa e ausência de balistoconídio, algumas espécies podem ainda apresentar-se capsuladas (FELL; STATZELL-TALLMAN, 2000 apud ALMEIDA, 2005).

Os agentes terapêuticos atualmente utilizados para o tratamento das infecções fúngicas, compreendem especialmente os polienos como anfotericina B e a nistatina; e derivados azóolicos como fluconazol e cetoconazol (RANG; DALE, 2007). Os antifúngicos poliênicos alteram a função da membrana celular dos fungos, estes fármacos ligam-se irreversivelmente ao ergosterol da membrana celular dos fungos, rompendo-os ou alterando a sua permeabilidade. Os compostos azólicos atuam mediante a inibição da enzima lanosterol 14- $\alpha$ demetilase no complexo citocromo P-450 dos fungos. O resultado é a inibição da conversão de lanosterol em ergosterol, com a depleção conseguinte de ergosterol, acumulação de precursores e perda da integridade da membrana fúngica (SANTOS JÚNIOR, 2005).

Uma vez conhecido os agentes terapêuticos utilizados no tratamento de fungemias oriundas de leveduroses, é importante ressaltar que o grau de resistência dos fungos aos fármacos antifúngicos vem sendo uma crescente. Fora visto que as leveduras Rhodotorula spp. apresentam maiores resistência ao fármaco Fluconazol (RICHARDSON; LASS-FLOR; 2008).

Devido à emergente resistência dos fungos aos antifúngicos clássicos e visando encontrar novas substâncias alternativas, nos últimos anos tem sido amplamente estudada a atividade antimicrobiana de diversas plantas e seus metabólitos isolados (CARMO et al., 2008; MOREIRA et al., 2010; MICELI; DÍAZ; LEE, 2011).

\section{Sida santaremnensis H. Monteiro (Malvaceae)}

A família Malvaceae encontra-se presente em quase todas as partes do mundo, sendo mais abundante nas regiões tropicais da América do Sul (HEYWOOD, 1993). É composta por 243 gêneros e 4.225 espécies (ESTEVENS, 2003 apud COSTA et al., 2007a). Quanto aos gêneros mais numerosos vistos na família Malvaceae encontram-se Hibiscus (300), Pavonia (270), Sida (200), Abutilon (100), Nototriche (100), Cristaria (75) e Gossypium (40) (HALL, 2002; ESTEVENS, 2003 apud COSTA et al. 2007a; ESTEVES, 2006).

O gênero Sida apresenta ampla distribuição neotropical com várias espécies bem representadas nas Américas. No Brasil este gênero possui muitos representantes nas regiões Nordeste e Sul, e em menor proporção, nas regiões Norte, Centro-Oeste e Sudeste (BARACHO, 1998 apud MELO e COSTA, 2011).

As investigações fitoquímicas realizadas para espécies da família Malvaceae, evidenciaram que estas espécies são ricas em ácidos graxos, óleos essenciais, sesquiterpenóides, esteróides, triterpenos, compostos fenólicos, flavonóides, entre muitos outros compostos (SILVA et al., 2006; COSTA et al., 2007b; SILVA et al., 2010).

Por apresentarem diferentes propriedades terapêuticas e relatos de uso, as plantas do gênero Sida, vêm sendo amplamente estudadas. No Brasil, a espécie Sida cordifolia, é usada na medicina popular para o tratamento de estomatites, da asma e de congestão nasal (MOURA, 2010). Enquanto outras espécies deste gênero destacam-se por sua utilização como adstringentes, antídotos para as peçonhas do escorpião e cobra, agentes antituberculosos, febrífugos, antiinflamatórios (GUNATILAKA et al., 1980; VENKATESH et al., 1999 apud MOURA, 2010). O extrato etanólico obtido de outra espécie do gênero Sida (Sida acuta) apresentou atividade analgésica quando realizado teste da placa quente (DIWAN; KANTH, 1999 apud MOURA, 2010). A atividade antimicrobiana do gênero Sida já foi descrita para as espécies Sida acuta Burn. e Sida rhombifolia L. (LOPES et al., 2008).

A espécie Sida santaremensis H. Monteiro (figura 2, p.19) ocorre em quase todo o Brasil à exceção da Região Sul, onde não se tem registro (BOVINI, 2010). Apesar de sua ampla distribuição, não há relato da utilização dessa espécie na medicina popular. Contudo, verificando a variedade de compostos naturais, propriedades farmacológicas e relatos de uso descritos para espécies da família Malvaceae, notadamente no gênero Sida, e visando contribuir para o desenvolvimento de novos antifúngicos, é oportuno avaliar a sensibilidade e cepas de Rhodotorula spp. frente ao extrato e frações da $S$. santaremnensis.

\section{MATERIAL E MÉTODOS}

\section{Local de trabalho}

O trabalho foi realizado no Laboratório de Microbiologia da Unidade Acadêmica de Saúde (UAS), do Centro de Educação e Saúde (CES), da Universidade Federal de Campina Grande (UFCG).

\section{Material vegetal}

As partes aéreas de Sida santaremnensis H. Monteiro foram coletadas no Parque Piauí (Teresina-PI) em abril de 
2007. A identificação botânica foi realizada pela Botânica Dr $^{\mathrm{a}}$. Gardene Maria de Sousa, sendo uma exsicata depositada no Herbário Graziella Barroso sob o código 21867, na Universidade Federal do Piauí (UFPI) em Teresina-PI.

Os extratos e frações da Sida santaremnensis foram cedidos pela Dra ${ }^{\mathrm{a}}$. Danielly Albuquerque da Costa da Universidade Federal de Campina Grande (UFCG).

\section{Armazenamento}

Os produtos permaneceram armazenados em um frasco âmbar e mantidos sob refrigeração, a uma temperatura inferior a $4^{\circ} \mathrm{C}$, no Laboratório de Microbiologia. As soluções nas diferentes concentrações foram preparadas no momento da execução dos ensaios, dissolvendo-os primeiramente em DMSO (dimetilsulfóxido) e utilizando água destilada estéril para alcançar a concentração inicial $2048 \mu \mathrm{g} / \mathrm{mL}$. A partir desta, foram feitas diluições seriadas em razão de dois para alcançar concentrações menores dos produtos a serem testados.

\section{Produtos controle}

$\mathrm{O}$ antifúngico utilizado como controle positivo foi a anfotericina B $(100 \mu \mathrm{g} / \mathrm{mL})$, obtida da Sigma-Aldrich ${ }^{\circledR}$.

\section{Fungos}

Nos ensaios de atividade antifúngica foram utilizadas doze cepas de Rhodotorula spp. cedidas pela Dra ${ }^{\text {a }}$ Edeltrudes de Oliveira Lima do laboratório de Micologia Clínica do Departamento de Ciências Farmacêuticas do Centro de Ciências da Saúde da Universidade Federal da Paraíba (UFPB).

\section{Meio de cultura}

O meio de cultura utilizado no ensaio para avaliação da atividade antifúngica foi o Caldo Sabouraud Dextrost.3 (CSD) adquirido da Difco ${ }^{\circledR}$, preparados de acordo com as instruções do fabricante. Para conservação das cepas e preparação do inóculo utilizou-se o Agar Sabouraud Dextrose (ASD), também adquirido da Difco ${ }^{\circledR}$. Os meios foram solubilizados com água destilada e esterilizados em autoclave, a $121^{\circ} \mathrm{C}$ por 15 minutos.

\section{Inóculo}

$\mathrm{Na}$ preparação do inóculo, os fungos foram primeiramente cultivados em meio ASD inclinado a $37^{\circ} \mathrm{C}$ por 24 horas (overnight). Inicialmente foram preparadas suspensões dos microrganismos em tubos contendo salina estéril $(\mathrm{NaCl}$ a $0,85 \% \mathrm{p} / \mathrm{v})$. Em seguida, essas suspensões foram agitadas por 2 minutos com auxílio do aparelho Vortex. Após agitação, cada suspensão teve sua turbidez comparada e ajustada àquela apresentada pela suspensão de sulfato de bário do tubo 0,5 da escala McFarland, a qual corresponde a um inóculo de aproximadamente $10^{6}$ unidades formadoras de colônias por mililitro (UFC/mL) (HADACEK; GREGER, 2000; SAHIN et al., 2004; BARROS et al., 2006).

\section{Determinação da Concentração Inibitória Mínima (CIM)}

A determinação da CIM do extrato etanólico bruto e frações de Sida santaremnensis foi realizada pela técnica de microdiluição, utilizando placas de microtitulação contendo 96 cavidades com fundo em forma de "U" e em duplicata (SOUZA et al., 2007; MOREIRA et al., 2010). Em cada orifício da placa foi adicionado $100 \mu \mathrm{L}$ do meio líquido CSD duplamente concentrado.

Posteriormente, $100 \mu \mathrm{L}$ da solução dos extratos, também duplamente concentrado, foram dispensados nas cavidades da primeira linha da placa. E por meio de uma diluição seriada a uma razão de dois (1:2), foram obtidas concentrações de $2048 \mu \mathrm{g} / \mathrm{mL}$ até $64 \mu \mathrm{g} / \mathrm{mL}$ de modo que na primeira linha da placa se encontrará a maior concentração e na sexta, a menor concentração. Por fim, foram adicionados $10 \mu \mathrm{L}$ do inóculo de Rhodotorulla spp. nas cavidades. A determinação da CIM foi conduzida com aproximadamente 1$5 \times 10^{5} \mathrm{UFC} / \mathrm{mL}$ do microrganismo em cada cavidade.

Um controle de viabilidade do microrganismo foi realizado colocando-se nas cavidades $100 \mu \mathrm{L}$ do mesmo CSD duplamente concentrado, $100 \mu \mathrm{L}$ de água destilada estéril e $10 \mu \mathrm{L}$ do inóculo. Um controle de esterilidade também foi realizado, onde foi colocado $200 \mu \mathrm{L}$ do CSD em um orifício sem a suspensão dos fungos. As placas foram incubadas a $37^{\circ} \mathrm{C}$ por até 48 horas para ser realizada a leitura. Paralelamente, foi realizado o mesmo experimento com o antifúngico anfotericina $\mathrm{B}(100 \mu \mathrm{g} / \mathrm{mL})$ comumente utilizado na terapêutica clínica de infecções por Rhodotorula spp.

Os valores de CIM foram determinados pela análise visual da inibição do crescimento em cada cavidade, comparando com o controle (ausente de drogas). Define-se a CIM para os produtos testados como a menor concentração capaz de inibir $100 \%$ do crescimento fúngico verificado nos orifícios. Os ensaios foram realizados em duplicata e o resultado expresso pela média geométrica dos resultados obtidos.

\section{Determinação da Concentração Fungicida Mínima (CFM)}

Após realização da microdiluição para determinação da CIM dos produtos, alíquotas de $20 \mu \mathrm{L}$ do produto presente nas cavidades onde não apresentaram crescimento fúngico foram semeadas em placas com ASD, desprovidas de qualquer antifúngico. Todo o sistema foi incubado a $37^{\circ} \mathrm{C}$ por até 48 horas. Os ensaios foram realizados em duplicata e o resultado expresso pela média geométrica das CFM obtidas nos dois ensaios. A CFM foi definida como a menor concentração dos produtos testados onde a cepa teste não mostrou capacidade de crescimento, quando inoculada no meio de cultura isento de antifúngicos (KONEMAN et al., 1993; RASOOLI; ABYANEH, 2004).

\section{RESULTADOS E DISCUSSÃO}

Dentre as doze cepas de Rhodotorula spp. testadas, os resultados de ação antifúngica foram alcançados sobre três delas. A atividade antifúngica foi observada para o extrato etanólico bruto e as frações acetato de etila e diclorometânica. A concentração inibitória mínima, onde não se visualizou crescimento fúngico, esteve entre $2048 \mu \mathrm{g} / \mathrm{mL}$ e $512 \mu \mathrm{g} / \mathrm{mL}$ (ver tabela 1). Nenhum grau de sensibilidade às frações 
hexânica e hidroalcoólica foi observado para as cepas de Rhodotorulla spp. ensaiadas.
Nos ensaios realizados com o antifúngico controle (anfotericina B) não ocorreu crescimento das cepas de Rhodotorula spp.

Tabela 1: Concentração Inibitória Mínima (CIM) do extrato etanólico bruto e frações de Sida santaremnensis sobre cepas de Rhodotorula spp.

\begin{tabular}{|c|c|c|c|c|c|}
\hline \multicolumn{6}{|c|}{ Concentração Inibitória Mínima (CIM) - $\mu \mathrm{g} / \mathrm{mL}$} \\
\hline \multirow[b]{2}{*}{ Microrganismos } & \multicolumn{4}{|c|}{ Material testado } & \multirow[b]{2}{*}{ Hidroalcoólica } \\
\hline & Extrato etanólico bruto & Acetato de etila & Diclorometânica & Hexânica & \\
\hline LM 02 & $\mathrm{R}$ & $\mathbf{R}$ & $\mathrm{R}$ & $\mathrm{R}$ & $\mathrm{R}$ \\
\hline LM 05 & $\mathrm{R}$ & $\mathbf{R}$ & $\mathrm{R}$ & $\mathrm{R}$ & $\mathrm{R}$ \\
\hline LM 012 & $\mathrm{R}$ & $\mathbf{R}$ & $\mathrm{R}$ & $\mathrm{R}$ & $\mathrm{R}$ \\
\hline LM 014 & $\mathrm{R}$ & $\mathbf{R}$ & $\mathrm{R}$ & $\mathrm{R}$ & $\mathrm{R}$ \\
\hline LM 18 & 2048 & 512 & 1024 & $\mathrm{R}$ & $\mathrm{R}$ \\
\hline LM 25 & $\mathrm{R}$ & $\mathbf{R}$ & $\mathrm{R}$ & $\mathrm{R}$ & $\mathrm{R}$ \\
\hline LM 32 & $\mathrm{R}$ & $\mathbf{R}$ & $\mathrm{R}$ & $\mathrm{R}$ & $\mathrm{R}$ \\
\hline LM 081 & $\mathrm{R}$ & $\mathbf{R}$ & $\mathrm{R}$ & $\mathrm{R}$ & $\mathrm{R}$ \\
\hline LM 323 & 512 & 1024 & $\mathrm{R}$ & $\mathrm{R}$ & $\mathrm{R}$ \\
\hline LM 382 & 2048 & $\mathbf{R}$ & $\mathrm{R}$ & $\mathrm{R}$ & $\mathrm{R}$ \\
\hline LM 482 & $\mathrm{R}$ & $\mathbf{R}$ & $\mathrm{R}$ & $\mathrm{R}$ & $\mathrm{R}$ \\
\hline LM 888 & $\mathrm{R}$ & $\mathbf{R}$ & $\mathrm{R}$ & $\mathrm{R}$ & $\mathrm{R}$ \\
\hline
\end{tabular}

LM: Laboratório de Micologia

R: Resistente

O extrato etanólico bruto e as frações acetato de etila e diclorometânica da Sida santaremnensis, que demonstraram ação contra as cepas LM 18, LM 323 e LM 382, apresentaram também comportamento fungicida sobre as mesmas, uma vez que quando inoculadas alíquotas do sistema microrganismo, meio de cultura e extrato ou fração, em meio totalmente isento de antifúngico, não se visualizou crescimento algum do fungo.

Sida representa um dos gêneros mais numerosos da família Malvaceae. Espécies deste gênero são amplamente estudadas, sendo muitas delas já utilizadas com fins alimentícios e medicinais. Quanto a sua utilização na terapêutica, vários estudos farmacológicos foram elucidados com base no uso etnobotânico (ESTEVES, 2006; SILVA et al., 2006; COSTA et al., 2007 ${ }^{\mathrm{a}}$.

Com relação à atividade antimicrobiana deste gênero vários estudos vêm sendo desenvolvidos. Fora comprovada que as espécies Sida acuta Burm., Sida cordifolia L. e Sida rhomboidea Roxb. possuem significativa atividade antimicrobiana sobre bactérias, como Staphylococcus aureus, Pseudomonas aeruginosa, Escherichia coli, Bacilus subtilis e Mycobaterium phlei (KAROU et al., 2005; MEDEIROS et al., 2006; BRUGÉS; REZA, 2007; KAROU et al., 2007).

Anteriormente a este estudo, Silva et al. (2008) avaliaram a atividade do extrato etanólico das folhas de $S$. santaremnensis sobre bactérias gram-positivas e gramnegativas, verificando que o extrato não apresentou atividade sobre as bactérias E. coli, S. aureus e S. epidermidis. Mesmo possuindo inúmeras propriedades terapêuticas, os estudos sobre a atividade antimicrobiana para a espécie $S$. santaremnensis ainda são escassos, sendo esta a primeira vez que seu extrato etanólico bruto e frações foram testados contra leveduras do gênero Rhodotorula.

Os estudos fitoquímicos descritos para algumas espécies do gênero Sida mostraram que dentre os componentes químicos responsáveis pela atividade antimicrobiana destacam-se os alcalóides, flavonóides, polifenóis e saponinas (BRUGÉS; REZA, 2007; KAROU et al., 2007; LOPES et al., 2008), sendo estes correspondentes as mesmas classes de metabólitos secundários identificados na triagem fitoquímica da $S$. santaremnensis, como visualizado no quadro 1 (p.26). A análise destes dados permite sugerir que a inibição das cepas de Rhodotorula spp. possa estar relacionada com à presença destes constituintes encontrados no extrato etanólico bruto e nas fases diclorometânica e acetato de etila da espécie em estudo, uma vez que estes foram capazes de inibir as cepas de Rhodotorula spp. (Tabela 1, p.24).

Quadro 1- Triagem fitoquímica das partes aéreas de Sida santaremnensis (MELO; COSTA, 2011)

\begin{tabular}{|l|c|c|c|c|c|c|}
\hline \multicolumn{2}{|c|}{ PESQUISA } & \multicolumn{5}{c|}{ RESULTADO } \\
\hline $\begin{array}{l}\text { METABÓLITO } \\
\text { SECUNDÁRIO }\end{array}$ & TESTE & EEB & $\begin{array}{c}\text { FASE } \\
\text { HEX }\end{array}$ & $\begin{array}{c}\text { FASE } \\
\text { CH }_{2} \text { Cl}_{2}\end{array}$ & $\begin{array}{c}\text { FASE } \\
\text { ACOET }\end{array}$ & $\begin{array}{c}\text { FASE } \\
\text { ETOH:H }\end{array}$ \\
\hline Esteróides & Lieberman-Burchard & ++ & ++ & ++ & - & - \\
\hline Triterpenos & Lieberman-Burchard & + & + & + & ++ & - \\
\hline Flavonóides & Shinoda & + & - & + & ++ & +++ \\
\hline Taninos & Gelatina & + & - & - & ++ & +++ \\
\hline Alcalóides & Dragendorff & ++ & - & ++ & + & ++ \\
\hline Saponinas & Índice de Espuma & + & - & - & ++ & ++ \\
\hline
\end{tabular}

O sinal (+) indica presença e (-) ausência do constituinte químico. 
Os resultados da triagem de foram considerados positivos pela formação de precipitados e surgimento de coloração ou espuma, sendo classificados em fraco positivo $(+)$, moderado positivo (++) e forte positivo $(+++)$, pela intensificação destes e negativo (-) pela ausência dos mesmos. Nessa triagem da S. santaremnensis realizada por Melo e Costa (2011) observou-se que a fase hexânica (fase em que ocorreu resistência de todas as cepas de Rhodotorula spp.) não apresentou alcalóides, flavonóides ou saponinas, levando a sugestão de que a inibição das cepas é provavelmente oriunda de tais metabólitos. No caso das fases acetatoetílica e hidroalcoólica, seus constituintes químicos apresentam-se glicosilados, e a esses tipos de compostos geralmente não se atribui atividade antimicrobiana.

Percebeu-se neste estudo que as cepas de Rhodotorula spp. evidenciaram mais resistência que sensibilidade aos produtos testados. Um estudo semelhante a este foi realizado confrontando o extrato etanólico bruto de Sida acuta e diversas bactérias, além de leveduras da espécie Candida albicans, sendo verificado que a maioria das bactérias foram sensíveis, porém o extrato não foi eficiente em inibir o crescimento de C. albicans. Esse achado de Oboh; Akerele e Obasuyi (2007) corrobora nossos resultados, levantando outra hipótese que justifique essa resistência, onde os fungos ou especificamente as leveduras possuam mecanismos próprios de resistência aos compostos ativos dos extratos de $S$. santaremnensis.

De acordo com Sartoratto et al. (2004) uma substância para possuir uma forte atividade antimicrobiana deve apresentar inibição nas faixas de 50 a $500 \mu \mathrm{g} / \mathrm{mL}$, uma atividade moderada de 600 a $1500 \mu \mathrm{g} / \mathrm{mL}$ e fraca atividade substâncias acima de $1500 \mu \mathrm{g} / \mathrm{mL}$; sendo assim, um bom candidato a antifúngico deve promover a inibição da cepa testada em baixas concentrações, possuindo uma forte atividade. Embora algumas cepas tenham apresentado sensibilidade ao extrato etanólico bruto e as frações acetato de etila e diclorometânica, esta faixa situou-se entre 512 a 2048 $\mu \mathrm{g} / \mathrm{mL}$, ratificando a ideia da inviabilidade de se indicar num futuro $S$. santaremnensis contra infecções produzidas por Rhodotorulla spp.

\section{CONCLUSÃO}

Conclui-se que a grande maioria das cepas da levedura Rhodotorula spp. não foi sensível ao extrato etanólico bruto e frações de $S$. santaremnensis. As concentrações em que se obteve inibição, não são consideradas adequadas para o desenvolvimento de um novo fármaco. Permitindo-se inferir que no futuro, quando se buscar desenvolver algum medicamento antimicrobiano a partir desta espécie vegetal, este, certamente será contraindicado para o tratamento de infecções por Rhodotorulla spp. especialmente, devido ao grau de resistência observado neste estudo.

\section{REFERÊNCIAS BIBLIOGRÁFICAS}

AGÊNCIA NACIONAL DE VIGILÂNCIA SANITÁRIA. Detecção e identificação dos fungos de importância médica. MóduloVII., 24 p. 2004. Disponível em: <http://www.anvisa.gov.br/servicosaude/microbiologia/ mod_7_2004.pdf $>$. Acesso em: 12 nov. 2011.

ALECRIM, E.; TEIXEIRA, H. Fungos anemófilos da Cidade do Recife - Pernambuco Brasil. An Fac Med Univ Fed Pernamb. v. 18 p. 269-74, 1958. apud LIMA, A. L. H. et al. Estudo laboratorial das micoses oculares e fatores associados às ceratites. Arquivo Brasileiro de Oftalmologia, v. 68, n.1, p. 21-7, 2005.

ALMEIDA, G. M. D. Rhodotorula spp, isolados de hemocultura no Hospital das Clínicas da Faculdade de Medicina de São Paulo, características clínicas e microbiológicas. Tese (Doutorado), Faculdade de Medicina da Universidade de São Paulo, 139 f. 2005.

ALMEIDA, S. R. Micologia. $1^{\mathrm{a}}$ Edição Rio de Janeiro: Guanabara Koogan. 2008, 161p.

ARCANJO, D. D. R.; OLIVEIRA, N. N. P. M.; FERREIRAFILHO, E. S.; COSTA, D. A.; CHAVES, M. H.; BORGES, A. C. R.; OLIVEIRA, A. P.; OLIVEIRA, R. C. Vasorelaxant response induced by Sida santaremnensis $\mathrm{H}$. Monteiro ethanol extract on rat superior mesenteric artery. African Journal of Biotechnology. v. 10 n.65, p. 14587-14597, 2011.

BARACHO, G. S.: Dissertação de Mestrado, Universidade Federal da Paraíba, Brasil, 1998 apud MELO, J. F. S.; COSTA, D. A. Estudo fitoquímico de Sida santaremnensis H. Monteiro (Malvaceae) como fonte de princípios ativos e desenvolvimento de novos fármacos. In: VIII Congresso de Iniciação Científica da Universidade Federal De Campina Grande, 2011. Disponível em: < $\quad$ http://pesquisa .ufcg.edu.br/anais/2011/>, acesso em 20, mar.2012, $15: 30: 25$

BARROS, M. E. S; SANTOS, D. A.; HAMDAN, J. S. In vitro methods for antifungal susceptibility testing of Trichophyton spp. Mycological Research, v. 110, n. 11, p. 1355-1360, 2006.

BOVINI, M.G. Malvaceae s.str. na Reserva Rio das Pedras, Mangaratiba, Rio de Janeiro, Brasil. Rodriguésia, v.61 n. 2 p. 289-301, 2010.

BRUGÉS, K.; REZA, M. T. R. Evaluación preliminar de toxicidad, genotoxicidad y actividad antimicrobiana de Sida rhombifolia L. Revista Colombiana de Biotecnologia, v. 9, n. 1, p. 5-13, 2007.

CARMO, E. S.; LIMA, E. O.; SOUZA, E. L.; SOUSA, F. B. Effect of Cinnamomum zeylanicum Blume essential oil on the growth and morphogenesis of some potentially pathogenic Aspergillus species. Brazilian Journal of Microbiology, v.39, p. 91-97, 2008.

CHANDRASEKARA, A. I.; SRYANI, H. T. B. 1980. Studies on Medicinal Plants of Sri Lanka III: Pharmacologically Important Alkaloids of some Sida Species. Planta Medica. 39, p. 66-72. apud MOURA, W. R. A Tese (Doutorado) Universidade Federal do Piauí, 69 f. 2010. 
COSTA C. D. F.; HERCULANO E. A.; ARCANJO D.D.R.; OLIVEIRA R.C.M.; OLIVEIRA A.P.; RIBEIRO, E. A. N. Avaliação dos efeitos cardiovasculares induzidos pelo extrato etanólico de Sida Santaremnensis. In: X Congresso Italo-Latinoamericano de Etnomedicina, 2011, Fortaleza, CE, Anais eletrônicos... Disponível em: $<$ http://www .silae.it/docs/Atti_XX_Silae_Fortaleza.pdf $>$. Acesso em: 12 nov. 2011.

COSTA, D. A.; SILVA, D. A.; CAVALCANTI, A. C.; MEDEIROS, M. A. A.; LIMA, J. T.; CAVALCANTE, J. M. S.; AGRA, M. F.; SOUZA, M. F. V. Chemical constituents from Bakeridesia pickelii Monteiro (Malvaceae) and the relaxant activity of kaempferol-3-o$\beta$-d-(6"-E-p-coumaroyl) glucopyranoside on guinea-pig ileum. Química Nova, v. 30, n. 4, p. 901-903, 2007 b.

COSTA, M. P.; MAGALhÃES, N. S. S.; GOMES, F. E. S.; MACIEL, M. A. M. Uma revisão das atividades biológicas da trans-desidrocrotonina, um produto natural obtido de Croton cajucara. Revista Brasileira de Farmacognosia. v. 17, n. 2, p. 275-286, 2007a.

ESTEVES, G. L. Flora da reserva Ducke, Amazonas, Brasil: Malvaceae. Rodriguésia, v. 57, n. 2, p. 205-206, 2006.

HADACEK, F.; GREGER, H. Testing of antifungical natural products: methodologies, comparability of results and assay choise. Phytochemical Analysis, v.11, p. 137-147, 2000.

HALL, C. H.; SYTSMA, K. J.; ILTIS, H. H. Phylogeny of Capparaceae and Brassicaceae based on chloroplast sequence data. American Journal of Botany. v. 89, p. $1826-1842,2002$.

HEYWOOD, V. H . Flowering plants of world. B. T. London: Batsford Ltd, 1993.

KANTH V. R, DIWAN, P. V. Analgesic, antiinflammatory and hypoglycaemic activities of Sida cordifolia in rats. Phytother Res.v 13, p 75-7, 2000. apud MOURA, W. R. A. Tese (Doutorado) Universidade Federal do Piauí, 69 f. 2010 .

KAROU, S. D., NADEMBEGA, M. C. W.; ILBOUDO, D. P.; OUERMI, D.; GBEASSOR, M.; SOUZA, C.; SIMPORE, J. Sida acuta Burm. f.: a medicinal plant with numerous potencies. African Journal of Biotechnology, v. 6, n. 25, p. 2953-2959, 2007.

KAROU, S. D.; SAVADOGO, A.; CANINI, A.; YAMEOGO, S.; MONTESANO, C.; JACQUES SIMPORE, J.; COLIZZI, V.; TRAORE, A. S. Antibacterial activity of alkaloids from Sida acuta. African Journal of Biotechnology, v. 4, n.12, p. 14521457, 2005.

KONEMAN E. W.; ALLEN, S. D.; DOWEL-JÚNIOR, V. R.; SAMERS, H. M.; Diagnóstico Microbiológico. Texto Atlas. Editora Médica Panamericana. 2. ed. 1993. p. 452-485.
LACAZ, C. S.; PORTO. E.; MARTINS, J. E. C.; HEINSVACCARI, E. M.; MELO, N. T. Tratado de Micologia Médica, 10ª Edição. São Paulo: Sarvier, 2002, 1104p.

LEAL, R. S. Estudo etnofamacológico e fitoquímico das espécies medicinais Cleome Espinosa Jacq, Pavonia marians Moric e Croton cajucara Benth. Tese (Doutorado), Universidade Federal do Rio Grande do Norte, 190 f. 2008.

LIMA, A. L. H.; FORSETO, A.; DUPRAT, J. P.; ANDRADE, A.; SOUZA, L. B.; GODOY, P.; FREITAS, D. Estudo laboratorial das micoses oculares e fatores associados às ceratites. Arq. Bras. Oftalmol. v. 68 , n. 1, p. 21-7, 2005.

LOPES, S. S.; MENOR, J. C. A. S.; ARCANJO, D. D. R.; OLIVEIRA, R. C. M.; CHAVES, M. H.; COELHO, L. F. L.; SOARES, M. J. S. Avaliação da atividade antibacteriana de Sida santaremnensis, Monteiro. In: III Reunião Regional, Federação de Sociedades de Biologia Experimental, Fortaleza, CE. Anais eletrônicos. Disponível em: <http://www.fesbe.org.br/regional2008/?resumos/36.079 >. Acesso em: 30 out. 2011.

MEDEIROS, I. A.; SANTOS, M. R. V.; NASCIMENTO, N. M. S.; DUARTE, J.C. Cardiovascular effects of Sida cordifolia leaves extract in rats. Fitoterapia. v. 77, n.1, p. 19-27, 2006.

MELO, J. F. S.; COSTA, D. A. Estudo fitoquímico de Sida santaremnensis H. Monteiro (Malvaceae) como fonte de princípios ativos e desenvolvimento de novos fármacos. In: VIII Congresso de Iniciação Científica da Universidade Federal De Campina Grande, 2011. Disponível em: < http://pesquisa.ufcg.edu.br/anais/2011/>, acesso em 20, mar.2012.

MENDES, J. F.; NASCENTE, P. S.; SANTIN, R.; FEIJÓ, BUENO, A. M.; LUND, R. G.; MEIRELES, M. C. A. Isolamento de Rhodotorula sp. de mãos de profissionais da saúde. In: XVII Congresso de Iniciação Científica e X Enpos, 2008, Pelotas. Anais eletrônicos. Pelotas: UFPEL, 2008. Disponível em:<http://www.ufpel.edu.br/cic/2008/cd/pages/pdf/CA/ CA_01167.pdf >. Acesso em: 10 fev. 2012.

MENDES, R. M. B.; FIGUEIREDO, K. A.; LOPES, L. S.; PEREIRA, S. S.; OLIVEIRA, A. P. COSTA, D. A.; CHAVES, M. H.; ALMEIDA, F. R.C. Estudo do efeito antinociceptivo de Sida santaremnensis (Malvaceae). In: $40^{\circ}$ Congresso Brasileiro de Farmacologia e Terapêutica Experimental, 2008, Águas de Lindóia, SP. Anais eletrônicos. Disponível em: <http://asp.sbfte .org.br /pub/media/Setor07_2008.pdf >. Acesso em: 28 jan. 2012.

MICELI, M. H.; DÍAZ, J. A.; LEE, S. A. Emerging opportunistic yeast infections. Lancet Infectious Diseases, v.11, p. 142-51, 2011. 
MOREIRA, A. C. P.; LIMA, E. O.; WANDERLEY, P. A.; CARMO, E. S.; SOUZA, E. L. Chemical composition and antifungal activity of Hyptis suaveolens (L.) poit leaves essential oil against Aspergillus species. Brazilian Journal of Microbiology, v. 41, p. 28-33, 2010.

MOURA, W. R. A. Ensaio farmacologico das atividades antiinflamatória, citotoxicidade e toxicidade aguda da Copaifera luetzelburgii, HARM E Sida santaremnensis, MONTEIRO. Tese (Doutorado) Universidade Federal do Piauí, 69 f. 2010.

NEUFELD, P. M. Manual de micologia médica, técnicas básicas para diagnóstico. Rio de Janeiro: PNCQ, 1999, $230 \mathrm{p}$.

OBOH, I. E.; AKERELE, J.O.; OBASUY, O. Antimicrobial activity of the ethanol extract of the aerial parts of sida acuta burm.f. (malvaceae).Tropical Journal of Pharmaceutical Research, v. 6, n. 4, p. 809-813, 2007.

OLIVEIRA, E. T.; BRITO, C. A.; OLIVEIRA, M. R. C.; CHAVES, M. H.; OLIVEIRA, F. A. Atividade antiedematogênica da Sida santaremnensis H. Monteiro (Malvaceae) em modelos animais. In: $40^{\circ}$ Congresso Brasileiro de Farmacologia e Terapêutica Experimental, 2008, Águas de Lindóia, SP. Anais eletrônicos. Disponível

em:<http://asp.sbfte.org.br/pub/media/Setor05_2008.pdf $>$. Acesso em: 31 jan. 2012.

OLIVEIRA, N. N. P. M.; FILHO, E. S. F.; ARCANJO, D. D. R.; NUNES, A. F.; COSTA, F. S.; CHAVES, M. H.; OLIVEIRA, A. P.; OLIVEIRA, R. D. C. M. Calcium and potassium channels involvement on vasorelaxation of Sida santaremnensis H. Monteiro (Malvaceae)... In: XXVI Reunião Regional, Federação de Sociedades de Biologia Experimental, Rio de Janeiro, RJ. Anais eletrônicos. Disponível em: <http://www.fesbe.org.br/fesbe2011/programa/programa _fesbe2011_PAG01_324.pdf.> Acesso em: 21 nov. 2011.

RANG, H. P et al. Farmacologia. $6^{\text {a }}$ Edição, Rio de Janeiro: Elsevier, 2007, 829 p.

RASOOLI, I.; ABYANEH, M. R. Inhibitory effects of thyme oils on growth and aflatoxin production by Aspergillus parasiticus. Food Control, v. 15, n. 6, p. 479-483, 2004.

RICHARDSON, M.; LASS-FLOR, C. Changing epidemiology of systemic fungal infections. Journal Compilation, European Society of Clinical Microbiology and Infectious Diseases, Aústria., v. 14. Suplemento 4, 2008.

RUIZ, N. C.; ZORRILA, A. F. C. Aislamiento de Rhodotorula $s p$. en un paciente críticamente enfermo. Descripción de un caso y revisión de la literatura. Infectio, Revista de la Associación Colombiana de Infectología , Colombia., v. 5, n. 3, 2001.
SAHIN, F.; GULLUCE, M.; DAFERERA, D.; SOKMEN, A.; POLISSIOU, M.; AGAR, G.; OZER, H.; Biological activities of the essential oil and methanol extract of Origanum vulgare ssp vulgare in the Eastern Anatolia region of Turkey. Food Control, v. 15, n. 7, p. 549-557, 2004.

SANTOS, JR. I. D.; SOUZA, I. A. M.; BORGES, R. G.; SOUZA, L. B. S.; SANTANA, W. J.; COUTINHO, H. D. M. Características gerais da ação, do tratamento e da resistência fúngica ao fluconazol. Scientia Medica, Porto Alegre, RS., v. 15, n. 3, jul./set. 2005.

SARTORATTO, A.; MACHADO, A. L. M.; DELARMELINA, C.; FIGUEIRA, G. M.; DUARTE, M. C. T.; REHDER, V. L. G. Composition and antimicrobial activity of essential oils from aromatic plants used in Brazil. Brazilian Journal of Microbiology, v. 35, p. 275-280, 2004.

SIDRIM, J. J. C.; ROCHA, M. F. G. Micologia médica, a luz de autores contemporâneos. $1^{\mathrm{a}}$ Edição, Rio de Janeiro: Guanabara Koogan. 2004, [reimp] 2010, 388p.

SILVA, A.C.O et al. An approach to chemotaxonomy to the fatty acid content of some Malvaceae species. Biochemical Systematics and Ecology, v. 38 , p. 10351038, 2010.

SILVA, R. L.; MELO, G. B.; MELO, V. A.; ANTONIOLLI, A. R.; MICHELLONES, P. R. T.; ZULOCOT, S.; PICINATO, M. A. N. C.; MOTA, C. A.; SILVA, O. C. Effect of the aqueous extract of Sida cordifolia on liver regeneration after partial hepatectomy. Acta Cirúrgica Brasileira, v. 21, n. 1, p. 37-39, 2006.

SILVIA, F. V.; CARVALHO, K. I. M.; PEREIRA, S. S.; COSTA, D. A.; CHAVES, M. H.; OLIVEIRA, F. A.; OLIVEIRA, R. C. M. Atividade antiedematogênica de Sida santaremnensis H. Monteiro (Malvaceae) em modelos animais. In: III Reunião Regional, Federação de Sociedades de Biologia Experimental, Fortaleza, CE. Anais eletrônicos. Disponível em: <http://www.fesbe.org.br /regional2008/? resumos/36.029>. Acesso em: 20 jan. 2012.

SOUZA, E. L.; STAMFORD, T. L. M.; LIMA, E. O.; TRAJANO, V. N. Effectiveness of Origanum vulgare L. essential oil to inhibit the growth of food spoiling yeasts. Food Control, v. 18 n. 5, p. 409-413, 2007.

TUON, F. F., COSTA, S. F. Rhodotorula infection. A systematic review of 128 cases from literature. Revista Iberoamericana de Micologia, São Paulo, SP., v. 25, p. 35-140, 2008.

VENKATESH, S.; REDDY, S. R.; SURESH, B.; RESSY, B. M.; RAMESH, M. 1999. Antinociceptive and Antiinflammatory Activity of Sida rhomboidea Leaves. Journal of Ethnopharmacology. 67, p. 229-232. apud MOURA, W. R. A. Tese (Doutorado) Universidade Federal do Piauí, 69 f. 2010. 